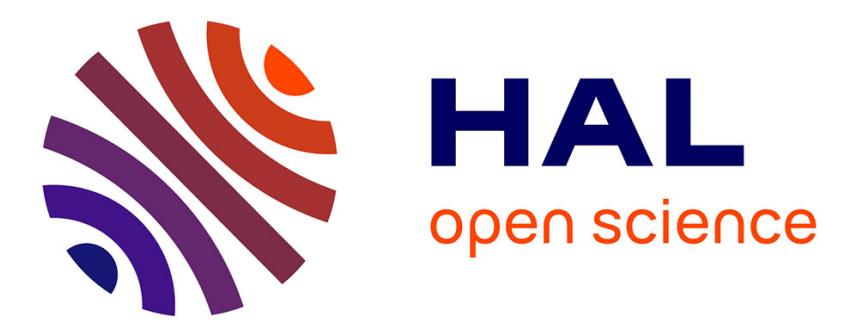

\title{
Local Porosity Measurement From Scanning Electron Microscopy Images in the Backscattered Electrons Mode.
}

Loïc Sorbier, Hedwige Poncet, Vincent Lecocq, Guillaume Maillet, Marwa Moula, Vincent Le Corre

\section{To cite this version:}

Loïc Sorbier, Hedwige Poncet, Vincent Lecocq, Guillaume Maillet, Marwa Moula, et al.. Local Porosity Measurement From Scanning Electron Microscopy Images in the Backscattered Electrons Mode.. Microscopy and Microanalysis, 2021, 27 (1), pp.20-27. 10.1017/S1431927620024782 . hal-03171782

\section{HAL Id: hal-03171782 \\ https://hal-ifp.archives-ouvertes.fr/hal-03171782}

Submitted on 17 Mar 2021

HAL is a multi-disciplinary open access archive for the deposit and dissemination of scientific research documents, whether they are published or not. The documents may come from teaching and research institutions in France or abroad, or from public or private research centers.
L'archive ouverte pluridisciplinaire HAL, est destinée au dépôt et à la diffusion de documents scientifiques de niveau recherche, publiés ou non, émanant des établissements d'enseignement et de recherche français ou étrangers, des laboratoires publics ou privés. 
Local porosity measurement from scanning electron microscopy images in backscattered electrons mode

Running title : Local porosity by SEM

Loïc Sorbier, Hedwige Poncet, Vincent Lecocq, Guillaume Maillet, Maroua Moula, Vincent Le Corre

IFP Energies nouvelles, Rond-point de l'échangeur de Solaize, BP 3, 69360 Solaize, France

Corresponding author : Loïc Sorbier, Tel : (+33) 4377029 69, Fax : (+33) 4377027 45, loic.sorbier@ifpen.fr 


\section{Abstract}

We propose a method to measure the local porosity of porous samples from scanning electron microscopy images in the backscattered electron mode. The porous samples are impregnated with a polymer resin and observed on polished cross-sections. Image intensities are calibrated with intensities from pure resin and the bulk phase. The calibration model is justified with Monte-Carlo simulations on perfectly homogeneous virtual samples. Uncertainties in measured porosity are given as function of uncertainties on physical properties of the resin and the bulk phase and on measured signals. The methodology is applied to a series of heterogeneous alumina catalyst supports with varying porosities. A good agreement is found between the averaged local porosity by scanning electron microscopy and global porosity determined by mercury intrusion porosimetry. The use of local porosity statistics allowed the quantitative characterization of the porosity fluctuations of these supports that appeared to be linked with their preparation parameters.

Key words : SEM, porosity, backscattered, Monte Carlo

\section{Introduction}

Porosity of materials is a key descriptor for their usage properties. In the field of heterogeneous catalysis, the porosity of supports controls both the transport (Kolitcheff et al., 2017) and mechanical properties (Wu et al., 2007). The more the support is porous, the more it is efficient for mass transport process but the weaker its mechanical properties are. Alumina catalyst supports are widely employed in several industrial processes such as 
reforming of naphta to increase the octane number of gasoline or hydrodesulfurization to remove sulfur from gas oil. Such alumina are porous solids in the form of millimeter sized spherical beads or extrudates with about $1 \mathrm{~mm}$ diameter and $1 \mathrm{~cm}$ length. These solids have pores in the 5-20 nm size range, specific surface area around $200 \mathrm{~m}^{2} / \mathrm{g}$ and porosity (volumic fraction of pores) around 0.7 . They are obtained by the dispersion of $2-10 \mathrm{~nm}$ sized crystallites of boehmite (aluminum oxy-hydroxide) in a aqueous paste that is calcinated to transform the boehmite crystalline phase into gamma alumina.

Whereas standard methods exist for global porosity assessment such as mercury intrusion porosimetry (MIP), nitrogen adsorption or helium pycnometry, few are able to give a local measure of the porosity. Yet, having local statistics of porosity is important for assessing spatial homogeneity of a sample or for mechanical strength studies as this strength is controlled by the most fragile fraction of the sample (Staub et al., 2016). Electron tomography allows to image catalyst supports at the nanometer scale but acquisition of tilted series, reconstruction and segmentation is a lengthy process. Moreover the small probed volumes (about $300 \mathrm{~nm}$ in size) raise questions about representativeness as alumina catalyst supports show heterogeneities up to few tenth of micrometers. X-ray computed tomography scanner is another possible choice to image resolved porosity (Taud et al., 2005) or compute unresolved porosity (Lin et al., 2016).

Scanning electron microscopy (SEM), with the help of Monte-Carlo simulations, may be employed to help the pore segmentation of non-impregnated porous sample prepared with a Focused Ion Beam (FIB) (Prill \& Schladitz, 2013). SEM has a limited spatial resolution, too large to be able to resolve the mesoporosity of alumina supports. However, SEM in the backscattered electron mode has been often employed to measure the local mean atomic 
number of multiphasic samples. Such procedure has been demonstrated on aluminum alloys (Ball \& McCartney, 1981), synthetic crystals and minerals (Herrmann \& Reimer, 1984), metallic alloys (Donovan et al., 2003), metals and minerals (Sánchez et al., 2012). The standard procedure to retrieve sample composition is to find a mixing model linking the sample atomic composition (either from mass or atomic concentration) to the backscattering yield.

Suppose that a sample is a homogeneous compound of $N$ distinct atoms with atomic number $Z_{i}$, mass concentration $c_{i}$ and atomic concentration $a_{i}$. A mixing rule relates the backscattering coefficient of the compound $\bar{\eta}$ with the sample composition and the backscattering yield of the pure elements $\eta_{i}$. Castaing has proposed a mixing rule based on the mass concentrations in the compound (Castaing, 1960):

$$
\bar{\eta}=\sum_{i=1}^{N} c_{i} \eta_{i}
$$

Most mixing models are reviewed by (Herrmann \& Reimer, 1984) and their conclusion is that the Castaing's relation leads to the better agreement with measurements. Howell and Boyde (Howell \& Boyde, 1998) have studied backscattering emission from low atomic number compounds and found no mixing model in agreement with their results. From measurements on isotope enriched samples, Donovan et al have shown that mass concentration mixing rules are irrelevant (Donovan et al., 2003). These authors proposed a modified electron fraction mixing rule based on an empirical exponent $x$ :

$$
\begin{aligned}
z_{i}^{(x)} & =\frac{a_{i} Z_{i}^{x}}{\sum_{j=1}^{N} a_{j} Z_{j}^{x}} \\
\bar{\eta}^{(x)} & =\sum_{i=1}^{N} z_{i}^{(x)} \eta_{i}
\end{aligned}
$$


They argued that backscattering is controlled by elastic scattering known to depend on atom nucleus charge which explain the dependence on electron fraction. By least square fitting of their experimental results on Au-Ag-Cu alloys, they obtain an empirical exponent $x=1.4$ that best fit their results. It is worth noticing that all the above works deals with the total backscattering coefficient and do not discuss the validity of the mixing rules for the backscattering signal captured by a annular detector.

The use of a mixing rule can be adapted to the problem of local porosity assessment. By local porosity measure, we mean the determination of the porosity in each pixel of a SEM image, this porosity being unresolved at the pixel scale. By embedding the porous sample in a resin, the local mean atomic number becomes related to the local concentration of resin and thus to the local porosity. The idea has been indeed proposed by Dang and Igarashi to characterize water content in concrete (Hoang \& Igarashi, 2013) and Moula et al. for the study of the mechanical damage of porous alumina supports (Moula et al., 2020). However these authors have not checked the validity of the mixing model employed to calculate the local porosity nor have given the measurement errors implied by their method.

The aim of this paper is thus to propose a robust and justified method to obtain the porosity of a mesoporous sample at a micrometer resolution associated with an uncertainty estimation. The method implies the preparation of the sample and of standards, their imaging by SEM, and the processing of the grey levels of the images.

The first section of the paper describes the porosity formula obtained from mass concentration or modified electron fraction mixing rules, the evaluation of uncertainties, the Monte Carlo simulations methodology, the shaping of supports and the sample preparation and imaging for SEM. Results are presented in a following section. The Monte Carlo results 
allows us to choose the appropriate mixing rule. The full method is applied to a series of alumina supports with varying mean porosities. The last section is devoted to the discussion of results followed by conclusions.

\section{Materials and Methods}

\section{Mass concentration mixing rule}

We suppose that the sample is a homogeneous mixture of a solid bulk phase of specific mass $\rho_{b}$ and a resin of specific mass $\rho_{r}$. The mass concentration of resin $c_{r}$ reads :

$$
c_{r}=\frac{\varepsilon \rho_{r}}{\varepsilon \rho_{r}+(1-\varepsilon) \rho_{b}}
$$

with $\varepsilon$ the porosity. The signal in backscattered electrons $\bar{\eta}$ of the mixture reads, according to the Castaing's relation:

$$
\bar{\eta}=\eta_{r} c_{r}+\eta_{b}\left(1-c_{r}\right)
$$

where $\eta_{r}$ and $\eta_{b}$ are the signals measured on pure resin and bulk phase respectively. Combining equations ( 3 ) and ( 4 ) leads to:

$$
\varepsilon=\frac{\rho_{b}\left(\eta_{b}-\bar{\eta}\right)}{\rho_{b}\left(\eta_{b}-\bar{\eta}\right)+\rho_{r}\left(\bar{\eta}-\eta_{r}\right)}
$$

If $\rho_{r}$ and $\rho_{b}$ are known, the measurement of $\bar{\eta}, \eta_{r}$ and $\eta_{b}$ allows the determination of $\varepsilon$ through equation ( 5 ).

\section{Modified electron fraction mixing rule}

Let $\mathcal{M}_{b}$ and $\mathcal{M}_{r}$ be the molar mass of the bulk phase and of the resin respectively and $\mathcal{V}_{b}=\mathcal{M}_{b} / \rho_{b}$ and $\mathcal{V}_{r}=\mathcal{M}_{r} / \rho_{r}$ the molar volumes of the bulk phase and the resin respectively. The porosity reads: 


$$
\varepsilon=\frac{\mathcal{V}_{r} a_{r}}{\mathcal{V}_{r} a_{r}+\mathcal{V}_{b}\left(1-a_{r}\right)}
$$

where $a_{r}$ is the molar concentration of the resin. According to the Donovan's relation (Donovan et al., 2003), the signal in backscattered electrons $\bar{\eta}$ of the mixture reads:

$$
\bar{\eta}=\eta_{r} \frac{a_{r} Z_{r}^{x}}{a_{r} Z_{r}^{x}+\left(1-a_{r}\right) Z_{b}^{x}}+\eta_{b} \frac{\left(1-a_{r}\right) Z_{b}^{x}}{a_{r} Z_{r}^{x}+\left(1-a_{r}\right) Z_{b}^{x}}
$$

where $Z_{b}$ and $Z_{r}$ are the modified atomic numbers of the bulk phase and the resin respectively and $x$ is the empirical exponent of Donovan's relation (Donovan et al., 2003). Combining equations ( 6 ) and ( 7 ) leads to:

$$
\varepsilon=\frac{\mathcal{V}_{r} Z_{b}^{x}\left(\eta_{b}-\bar{\eta}\right)}{\mathcal{V}_{r} Z_{b}^{x}\left(\eta_{b}-\bar{\eta}\right)+\mathcal{V}_{b} Z_{r}^{x}\left(\bar{\eta}-\eta_{r}\right)}
$$

By knowing $\mathcal{V}_{b}, \mathcal{V}_{r}, Z_{b}, Z_{r}$ and measuring $\eta_{b}, \eta_{r}$ and $\bar{\eta}$, it is possible to obtain the local porosity. Suppose that the signal is acquired with a 8 bits depth, that the resin leads to a signal $\eta_{r}=20$ and the bulk alumina phase to a signal $\eta_{b}=235$. Figure 1 reports the calibration curves linking the porosity with the measured signal from a mass concentration and a modified electron fraction mixing rule.

Standard procedure of uncertainty propagation applied to equation ( 8 ) leads to (see supplementary materials):

$$
\begin{gathered}
\frac{u_{\varepsilon}^{2}}{\varepsilon^{2}}=\varepsilon^{2}\left(\frac{\mathcal{v}_{b} Z_{r}^{x}}{\mathcal{V}_{r} Z_{b}^{x}}\right)^{2}\left[\left(\frac{\bar{\eta}-\eta_{r}}{\eta_{b}-\bar{\eta}}\right)^{2}\left(\frac{u_{v_{b}}{ }^{2}}{\mathcal{v}_{b}{ }^{2}}+\frac{u_{v_{r}}{ }^{2}}{\mathcal{v}_{r}{ }^{2}}+\frac{u_{z_{b}^{x^{2}}}}{Z_{b}^{x^{2}}}+\frac{u_{z_{r}^{x^{2}}}}{Z_{r}^{x^{2}}}\right)\right. \\
+\frac{1}{\left(\eta_{b}-\bar{\eta}\right)^{4}}\left[\eta_{b}^{2}\left(\bar{\eta}-\eta_{r}\right)^{2} \frac{u_{\eta_{b}}{ }^{2}}{\eta_{b}{ }^{2}}+\eta_{r}{ }^{2}\left(\eta_{b}-\bar{\eta}\right)^{2} \frac{u_{\eta_{r}}{ }^{2}}{\eta_{r}{ }^{2}}\right. \\
\left.\left.+\bar{\eta}^{2}\left(\eta_{b}-\eta_{r}\right)^{2} \frac{u_{\bar{\eta}}{ }^{2}}{\bar{\eta}^{2}}\right]\right]
\end{gathered}
$$


where $u_{Y}$ is the standard uncertainty of quantity $Y$. Inspection of equation ( 9 ) shows an indetermination for $\bar{\eta}=\eta_{b}$ otherwise said for $\varepsilon=0$. An alternative evaluation of $u_{\varepsilon}$ through an explicit expression for $f=1-\varepsilon$ allows to handle the case $\varepsilon=0$ (see supplementary materials). Now, it is interesting to examine the order of magnitude of the different terms of equation (9). With the assumptions that the resin leads to a signal $\eta_{r}=20$ and the bulk alumina phase to a signal $\eta_{b}=235$, for a signal $\bar{\eta}=142$, equation ( 8 ) gives $\varepsilon \approx 0.701$ which is the order of magnitude of the global porosity of mesoporous alumina supports. Using these hypothesis and the physical data of Table 1, we obtain:

$$
\begin{aligned}
& \frac{u_{\varepsilon}{ }^{2}}{\varepsilon^{2}} \approx 8,95 \times 10^{-2}\left(\frac{u_{v_{b}}{ }^{2}}{v_{b}{ }^{2}}+\frac{u_{v_{r}}{ }^{2}}{\mathcal{v}_{r}{ }^{2}}+\frac{u_{z_{b}{ }^{2}}}{Z_{b}^{x^{2}}}+\frac{u_{z_{r}^{x}}{ }^{2}}{Z_{r}^{x^{2}}}\right) \\
& +0,653 \frac{u_{\eta_{b}}{ }^{2}}{\eta_{b}{ }^{2}}+2,19 \times 10^{-3} \frac{u_{\eta_{r}}{ }^{2}}{\eta_{r}{ }^{2}}+0,731 \frac{u_{\bar{\eta}^{2}}}{\bar{\eta}^{2}}
\end{aligned}
$$

Two terms are important contributors to the uncertainty on $\varepsilon$. The first leading term is related to $\eta_{b}$. Yet, it is possible to measure $\eta_{b}$ with a very high accuracy as an average on a large number of pixels of an image of the bulk phase. The second leading term is related to $\bar{\eta}$ and cannot be averaged. The order of magnitude of the relative accuracy of $\varepsilon$ is then the same as the relative accuracy of $\bar{\eta}$.

Figure 2 reports the relative and absolute errors of porosity determined by equation ( 9 ) as function of porosity and relative error on the measurement of local backscattered signal.

\section{Evaluation of the accuracy of $\bar{\eta}$}

The accuracy of the measurement of $\bar{\eta}$ is due to the Poisson noise of the electron beam, the Poisson noise of the detected electrons and the electronic noises of pre-amplifier and amplifier of the detector. Fortunately, the noise may be evaluated from the noise observed 
on the standards (pure resin and bulk phase) acquired in the same conditions as the samples. The modeling of the acquisition process (see supplementary materials) leads to:

$$
u_{\bar{\eta}}^{2}=\alpha \bar{\eta}+\beta
$$

The $\alpha$ and $\beta$ parameters are then determined from the uncertainties on the standards. As the Gaussian distribution is the limiting form of the Poisson distribution for large numbers of detected electrons, the uncertainties $u_{\bar{\eta}}$ in the standards are calculated the following way. The histogram of an image of the standard is computed and this histogram is least-square fitted with a single Gaussian peak. Standard deviation of the fitted peak gives $u_{\bar{\eta}}$ either on resin or on the bulk phase are obtained and then allow to determine $\alpha$ and $\beta$. This same procedure allows also to obtain $\eta_{b}$ or $\eta_{r}$ as the mean of the fitted peak and $u_{\eta_{b}}$ and $u_{\eta_{r}}$ as the uncertainty in these parameters estimation.

\section{Monte Carlo simulations}

We wrote a program to simulate the total backscattering coefficient and the angular distribution of backscattered electrons of a planar, semi-infinite medium at normal incidence using the simulation package PENELOPE (Salvat et al., 2011; Sempau et al., 1997). PENELOPE parameters were set to proceed with analog simulations (single scattering model). Electron trajectories were simulated for a $15 \mathrm{keV}$ impinging energy until the electron kinetic energy reaches $100 \mathrm{eV}$ (lowest energy allowed by PENELOPE) or the electron leaves the material. For each simulation, $10^{6}$ primary electron trajectories were generated and all reported uncertainties of simulation in this work are for a 99.7\% confidence level $(3 \sigma)$. 21 virtual materials consisting of non-porous, perfect mixtures between alumina and PMMA resin were defined with resin concentration ranging from 0 (pure alumina) to $100 \%$ (pure resin). These materials define virtual porous materials with infinitely small pores and porosity 
ranging from 0 to 1 as calculated by equation ( 6 ). For each virtual material, the angular distribution of backscattered electrons is simulated within 100 classes equidistantly spaced in $\cos \alpha$ where $\alpha$ is the angle between the material's surface normal and the emerging direction of the backscattered electron. The geometry of the detector is annular with a 2.5 $\mathrm{mm}$ inner radius, $6 \mathrm{~mm}$ outer radius and a working distance of $3.87 \mathrm{~mm}$ as shown in Figure 3. After the simulation, the backscattering coefficient detected by the annular detector is computed by integrating this simulated angular distribution between the inner $\alpha_{i}$ and outer $\alpha_{o}$ collection angles.

\section{Catalyst supports shaping}

Six gamma alumina supports were prepared from the same boehmite powder with different conditions so to vary their porosity. The boehmite was synthesized by co-precipitation of an acidic and a basic precursors containing aluminium in water. After washing and drying, the powder was divided into six batches. Each batch was kneaded in a Brabender mixer. Kneading was performed by adding an acid solution of $\mathrm{HNO}_{3}$ (peptisation step of the powder to obtain a paste) and then an ammonia solution for the neutralization step. From batch 1 to batch 6 , the total amount of kneading energy was varied from $500 \mathrm{~J} / \mathrm{g}$ to $5000 \mathrm{~J} / \mathrm{g}$, with a constant couple of $10 \mathrm{~N} . \mathrm{m}$. This energy was varied by changing the kneading duration. As an example, batch B500 was kneaded during 20 min and B5000 during 200 min.

Support extrudates were then characterized by mercury intrusion porosimetry after a pretreatment $\left(523 \mathrm{~K}\right.$ for $2 \mathrm{~h}$ ). The grain density $\rho_{g}$ is obtained at a $0.2 \mathrm{MPa}$ intrusion pressure where mercury fills only intergrain porosity but not intragrain porosity. Expected uncertainty on $\rho_{g}$ measurement is $5 \%$ relative as obtained from repeated measurement on certified porous samples. The porosity $\varepsilon$ (void volume fraction) is obtained using: 


$$
\varepsilon=1-\frac{\rho_{g}}{\rho_{b}}
$$

With $\rho_{b}$ the specific mass of bulk gamma alumina.

\section{SEM imaging}

Extrudates were dried in oven one night at $50^{\circ} \mathrm{C}$. For each sample, five extrudates were stuck with mastic (Instant VULLER, Polyfilla) on a metallic ring. Each ring was put at the bottom of a separate aluminum cylindrical mold of $2.5 \mathrm{~cm}$ diameter and $10 \mathrm{~cm}$ height. A liquid monomer, methyl methacrylate (MMA, Aldrich) with a radical initiator for polymerization, azo-bis-isobutyronitrile (AZDN, Sigma-Aldrich) in powder form, were mixed. When the mix was homogeneous, it was introduced to fill about the half of each mold. The molds were placed in a vacuum chamber where primary vacuum was maintained for $30 \mathrm{~min}$. The vacuum chamber was then put at atmospheric conditions and the molds were placed in an autoclave (Autoclave France). The polymerization occurred in the autoclave for $36 \mathrm{~h}$ at $45^{\circ} \mathrm{C}$ under nitrogen pressure of $7 \mathrm{MPa}$. By impregnating the MMA monomer in the porosity and polymerizing it directly in the pores to obtain poly methyl methacrylate (PMMA) the impregnation should be homogeneous. Indeed, the monomer is a very small molecule compared to pore size which gives it a good accessibility to porosity. A good impregnation is mandatory for two reasons. On the one hand, a porous sample with pores much smaller than the electron range leads to the same backscattering coefficient of the corresponding massive sample since elastic cross sections do not depend on material density and inelastic cross-sections do depend on density only for very high energy electrons. On the other hand, larger pores will produce topographic contrast not related with the pore volume. 
The autoclave was then purged and opened. The aluminum molds were separated from the plastic part leading to a solid plastic cylinder of $2.5 \mathrm{~cm}$ diameter and $5 \mathrm{~cm}$ height. Each cylinder was cut with a rotating blade (Buehler, Isomet 1000) to remove the metallic ring and the mossy plastic in order to obtain a $2.5 \mathrm{~cm}$ diameter and about $1 \mathrm{~cm}$ high cylindrical stub. Each stub was then polished with water, at the beginning with SiC papers and finished with diamonds (Struers, EcoMet250/AutoMet250). A massive alumina, 99.9 \% purity (Goodfellow) was prepared the same way. A thin conductive carbon layer was then deposited (Emitech, K975X) on each stub.

Stubs were placed in the sample holder of a Zeiss SUPRA 40 SEM and imaged with an HDASB detector at $15 \mathrm{kV}$ and a beam current was estimated about $10 \mathrm{nA}$. Images were acquired in 8 bits depth with an indicated magnification of $\times 250$ at $1024 \times 768$ resolution leading to a pixel size of $446 \mathrm{~nm}$. The working distance was adjusted to optimize the intensity of the detected backscattered electron signal on an image of the massive alumina sample. The working distance was then kept constant to this optimum $(4.3 \mathrm{~mm})$ for samples and standards. Imaging at the optimal working distance does not only optimize the detected signal but also limits the influence of the precise positioning of the samples and standards (see supplementary materials). The pre-amplifier gain of the detector and the contrast and brightness were adjusted to maximize the separation of pure resin and bulk alumina mean grey levels while preventing saturation (pixels at grey levels 0 or 255) and minimizing standard deviation of grey levels on bulk alumina. Dwell time was set to $25.6 \mu$ s leading to $20.2 \mathrm{~s}$ per frame. These conditions were chosen as a compromise to minimize the image noise while keeping an acceptable acquisition time and limit the destruction of the resin due to radiation damages. These settings were kept constant for the acquisition of the whole set of images with the help of a dedicated software using the Zeiss application programming 
interface (API), allowing automatic acquisition of predefined zones on each sample and standards. To control for beam current stability, images on standards were taken at the beginning and at the end of the sequence. The total acquisition time of sample and standards images lasted about 15 min (for 5 images per extrudate and for 5 extrudates examined).

\section{Results}

\section{Mass concentration mixing rule}

Figure 4 (dashed line) compares the Monte Carlo simulation results with equation ( 5 ) for total and detected backscattering coefficient assuming a mass concentration mixing rule (Castaing's relation). There is a poor agreement for intermediate porosities.

\section{Electronic fraction mixing rule}

Figure 4 (dotted line) compares the Monte Carlo simulation results with equation ( 8 ) for total and detected backscattering coefficient assuming an electronic fraction mixing rule (Donovan's relation) with the empirical exponent $x=1.4$. Agreement is better than for the mass mixing rule but not yet satisfactory.

By least-square fitting of $x$ in equation ( 8 ) on the Monte Carlo simulation results, a very good agreement is found on the whole range of porosity. The results are reported in Figure 4 (solid line). Fitted values are $x=1.312 \pm 0.004$ and $x=1.319 \pm 0.007$ for total and detected backscattering coefficient respectively.

\section{Application to alumina catalyst supports}


Figure 5 reports representative SEM pictures of the samples. On each support, we observe spherical grains of 1-100 $\mu \mathrm{m}$ diameters of light grey level entrapped in a darker matrix. As grains and matrix are both made of the same material (gamma alumina), the difference in grey levels is due to a difference in porosity. The grains have a lower porosity than the matrix, thus they contains less resin and appear brighter. We can qualitatively observe that the preparation procedure has a significant influence on the homogeneity of the support. The higher the kneading energy is, the less numerous the dense grains are. It is not clear however which support has the highest mean porosity. Around 25 SEM images per sample were processed to retrieve the local porosity. An example of mean porosity image is showed in Figure 6. From these images, a mean porosity and a standard deviation on the whole set of images were computed for each sample. The results of textural characterization and mean porosity on 25 images of the samples are reported in Table 2 . The different kneading conditions leads to different porosities as measured by SEM and nitrogen adsorption. The correlation between porosities obtained by SEM and MIP is showed in Figure 7.

\section{Discussion}

Figure 1 shows that the relation between local porosity and backscattering coefficient is not linear in contradiction with the simple model employed by Dang (Hoang \& Igarashi, 2013). Inspection of Figure 2 indicates that a reasonable error on porosity is expected for high porosities and low-noise SEM images. Notice however that for small porosities $\varepsilon \ll 1$, the relative error diverges. It means that the porosity cannot be obtained with a reasonable accuracy on high density zones. Fortunately, these high density zones are very seldom observed on alumina supports. 
The Monte Carlo simulations clearly indicate that a mass concentration mixing rule based on the Castaing's relation is not appropriate to model the simulation results. A mixing rule based on electron fraction as proposed by Donovan et al. with the recommended empirical exponent $x=1.4$ better fits the simulation results. By least square fitting of the Monte Carlo simulation results, this kind of mixing rule leads to a very satisfactory agreement. It is worth noticing that this mixing rule is valid either for the total backscattering coefficient or for the backscattering coefficient as measured by an annular detector. The optimized empirical exponent is close to the exponent 1.35 , which makes the modified electron fraction mixing rule to be equivalent to the elastic scattering fraction mixing rule suggested by Armstrong (Armstrong, 1991).

The validity of the assumptions of the mixing rule for the alumina supports must be discussed. These supports have a mean pore size around $10 \mathrm{~nm}$. The electron interaction volume in the resin or the bulk alumina is in the micrometer range for $15 \mathrm{keV}$ electrons. The samples can therefore be considered homogeneous at the interaction volume scale. Some macropores with size bigger than pixel size $(0.45 \mu \mathrm{m})$ are sometimes observed. It is clear that in the borders of these zones, the assumption of a homogenous sample is not fulfilled. However, as these borders represent only a very small fraction of the observed surface, their influence on averaged quantities is negligible. Notice that for well resolved macropores (volume interaction entirely lying in the resin) the proposed method gives a correct porosity $(\varepsilon=0)$ with a very good accuracy. Now the validity of the method could be questioned if the resin impregnation is not complete and homogeneous. It is very improbable that samples with mean porosity around 0.7 contains occluded (closed) porosity where the monomer could not penetrate. As no grey level gradient is observed in SEM images at the extrudates scale, there is an experimental evidence that the resin impregnation is homogenous at the 
extrudate scale (millimeter scale). The resin impregnation has been checked by SEM below the micron scale from $300 \mathrm{~nm}$ slices of resin embedded samples cut with ultramicrotome. Some very rare unfilled pores were observed, mostly in rare large cracks (see supplementary materials). Their size is around the micron or more. The very rareness of these unfilled pores indicates that the average porosity extracted from SEM results is still representative. These pores are probably due to trapped gaseous methyl methacrylate bubbles. Fulfilling the validity of the mixing rule also constraint the method to high electron primary energies in order to have an interaction range much larger that the pore size (note that besides, composition contrast vanishes at low voltage) and medium magnifications in order to not oversample (pixel size larger than imaging resolution).

The validity of the methodology relies also on the availability of a stable microscope. The electron beam current have to be stable in an hour time as well as the detector electronics (amplifiers and analog to digital converter). Comparing the standards before and after the image acquisition is a safe procedure to reject unreliable data. Moreover, the methodology presented here supposes a perfectly linear response of the detection chain. We have not explored this cause of error.

The application of the method to a series of alumina supports gives quantitative insight to the influence of the preparation process on mean porosity and spatial heterogeneity of porosity. Mean porosities obtained by SEM are in agreement with the ones determined by MIP considered as a reference. The maximal absolute relative error is less than $5 \%$ and the relative root mean square error is $2.6 \%$. SEM allows us to add supplementary information on the heterogeneity of the samples. The increase of mean porosity with kneading energy is explained by the dispersion of the boehmite crystallites packed in dense, low porosity grains 
(high grey levels in Figure 5) into a higher porosity phase (low grey levels in Figure 5). This qualitative information could not have been obtained without the quantitative local measurement of the porosity. However, having these local porosities will allow to establish models for the prediction of mechanical and transport properties of the supports at the grain scale. For this purpose, porosities of the low and high density phases as well as resolved macropores can be easily obtained by the segmentation of the quantitative porosity images into three classes.

The proposed method has some limitations if to be extrapolated to other systems. First, the method is appropriate only for a biphasic (bulk/pore) medium. Other systems where the bulk phase has a varying chemistry are not suitable as local mean atomic number is no longer related to the local resin concentration alone. This forbids the method to important porous samples such as silica-alumina catalysts supports, concrete or rocks if these phases have composition gradients. The method requires the availability of standards samples. This is always possible for the resin even if the radiation damage of the resin limits the possible beam currents and scanning rates. Availability of a bulk standard depends on the studied system. As the sample must be seen as homogeneous at the interaction volume scale, it limits the applicability to mesoporous samples otherwise said to pore size in the $\mathrm{nm}$ scale. As previously said, the method is not appropriate if a significant closed pores fraction is present. The extrapolation of the same calibration curve to other system than alumina and PMMA resin is questionable. In principle, the modified electron fraction rule should well match the results, maybe with an exponent slightly different from the one reported here. Alumina has a mean atomic number close to the one of the resin, bulk materials with higher mean atomic number would give a larger contrast between the two materials and maybe reveal a failure of the mixing rule. However, as the calibration curve may be easily checked 
by Monte Carlo simulations, the validity of the method to any other particular system can be quickly checked.

\section{Conclusions}

We have proposed a method to measure the local porosity of porous samples by SEM. The method is based on the impregnation of the samples by a resin and the imaging of their cross sections in backscattered electrons mode. Monte Carlo simulations have shown that a mixing rule based on mass concentration is not appropriate to relate the backscattering coefficient and the sample composition. By fitting the simulation results with a mixing rule based on a modified electron fraction, a very satisfactory agreement is found. We have estimated the uncertainties of the method that are dominated by the measurement of the backscattered signal on the porous sample. The method has been applied to a series of alumina catalyst supports. The mean porosity determined by SEM is in agreement with the one measured by mercury intrusion porosimetry. The SEM measurements allows us to have a quantitative insight into the influence of preparation condition to the spatial heterogeneities of porosity. The validity of the method to other systems, while not demonstrated in this work, could be easily checked using Monte Carlo simulations.

\section{Acknowledgments}

S. Chalal is gratefully acknowledged for his help on the estimation of the solid angle of the detector and on automation of the SEM imaging. Authors thank M. Moreaud and P. Duchene for their contribution to least square fit of standard images histograms.

\section{References}


Armstrong, J.T. (1991) Quantitative Elemental Analysis of Individual Microparticles with Electron Beam Instruments. In Electron Probe Quantitation, Heinrich, K.F.J. \& Newbury, D.E. (Eds.), pp. 261-315. Boston, MA : Springer US.

Ball, M.D. \& McCartney, D.G. (1981) The measurement of atomic number and composition in an SEM using backscattered detectors. Journal of Microscopy 124, 57-68. https://doi.org/10.1111/j.1365-2818.1981.tb01305.x

Castaing, R. (1960) Electron Probe Microanalysis. Advances in Electronics and Electron Physics 13, 317-386. https://doi.org/10.1016/S0065-2539(08)60212-7

Donovan, J.J., Pingitore, N.E. \& Westphal, A. (2003) Compositional averaging of backscatter intensities in compounds. Microscopy and Microanalysis 9, 202-215. https://doi.org/10.1017/S1431927603030137

Herrmann, R. \& Reimer, L. (1984) Backscattering coefficient of multicomponent specimens. Scanning 6, 20-29. https://doi.org/10.1002/sca.4950060203

Hoang, D.G. \& Igarashi, S. (2013) Determination of water-cement ratios of hardened cement pastes based on the estimation of under-pixel porosity in backscattered electron images. International Journal of Structural Engineering 4, 4. https://doi.org/10.1504/IJSTRUCTE.2013.050760

Howell, P.G. \& Boyde, A. (1998) Monte Carlo simulation of electron backscattering from compounds with low mean atomic number. Scanning 20, 45-49. https://doi.org/10.1002/sca.1998.4950200107

Kolitcheff, S., Jolimaitre, E., Hugon, A., Verstraete, J., Carrette, P.-L. \& Tayakout-Fayolle, M. (2017) Tortuosity of mesoporous alumina catalyst supports: Influence of the pore network organization. Microporous and Mesoporous Materials 248, 91-98. https://doi.org/10.1016/i.micromeso.2017.04.010

Lin, Q., Al-Khulaifi, Y., Blunt, M.J. \& Bijeljic, B. (2016) Quantification of sub-resolution porosity in carbonate rocks by applying high-salinity contrast brine using X-ray microtomography differential imaging. Advances in Water Resources 96, 306-322. https://doi.org/10.1016/j.advwatres.2016.08.002

Moula, M., Meille, S., Le Corre, V. \& Chevalier, J. (2020) Mechanical characterization of meso-porous alumina by micro- and nano-indentation. Materials Today Communications 25, 101315. https://doi.org/10.1016/j.mtcomm.2020.101315

Prill, T. \& Schladitz, K. (2013) Simulation of FIB-SEM images for analysis of porous microstructures. Scanning 35, 189-195. https://doi.org/10.1002/sca.21047

Sánchez, E., Torres Deluigi, M. \& Castellano, G. (2012) Mean atomic number quantitative assessment in backscattered electron imaging. Microscopy and microanalysis 18, 1355-1361. https://doi.org/10.1017/S1431927612013566

Sempau, J., Acosta, E., Baro, J., Fernández-Varea, J.M. \& Salvat, F. (1997) An algorithm for Monte Carlo simulation of coupled electron-photon transport. Nuclear Instruments and Methods in Physics Research Section B: Beam Interactions with Materials and Atoms 132, 377-390. https://doi.org/10.1016/S0168-583X(97)00414-X 
Staub, D., Meille, S., Le Corre, V., Rouleau, L. \& Chevalier, J. (2016) Identification of a damage criterion of a highly porous alumina ceramic. Acta Materialia 107, 261-272.

https://doi.org/10.1016/i.actamat.2016.01.071

Taud, H., Martinez-Angeles, R., Parrot, J.F. \& Hernandez-Escobedo, L. (2005) Porosity estimation method by X-ray computed tomography. Journal of Petroleum Science and Engineering 47, 209217. https://doi.org/10.1016/j.petrol.2005.03.009

Wu, D., Zhou, J. \& Li, Y. (2007) Mechanical strength of solid catalysts: Recent developments and future prospects. AIChE Journal 53, 2618-2629. https://doi.org/10.1002/aic.11291 


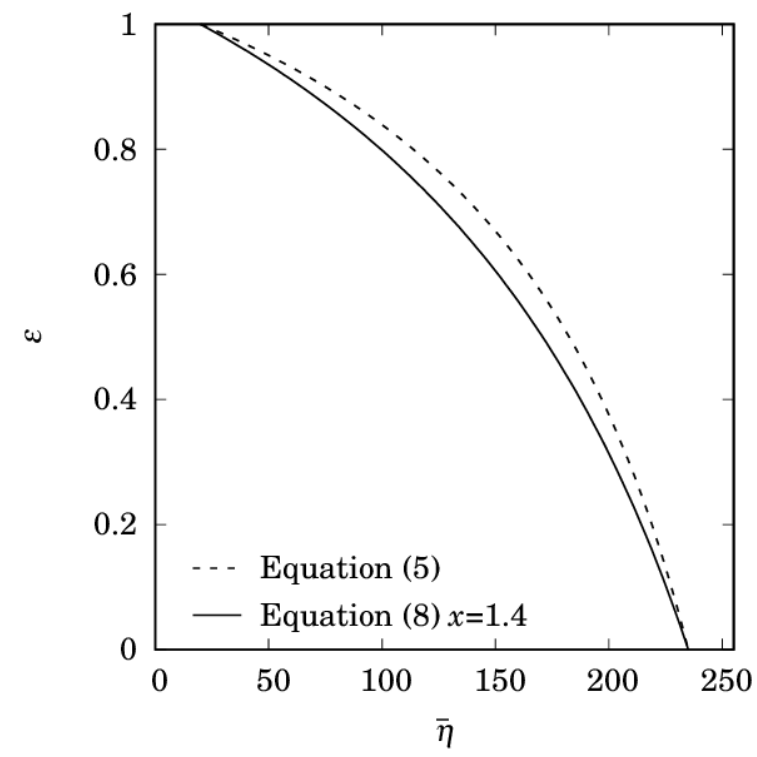

Figure 1 : Porosity as function of measured signal for $\eta_{b}=235, \eta_{r}=20$ and values of

Table 1.

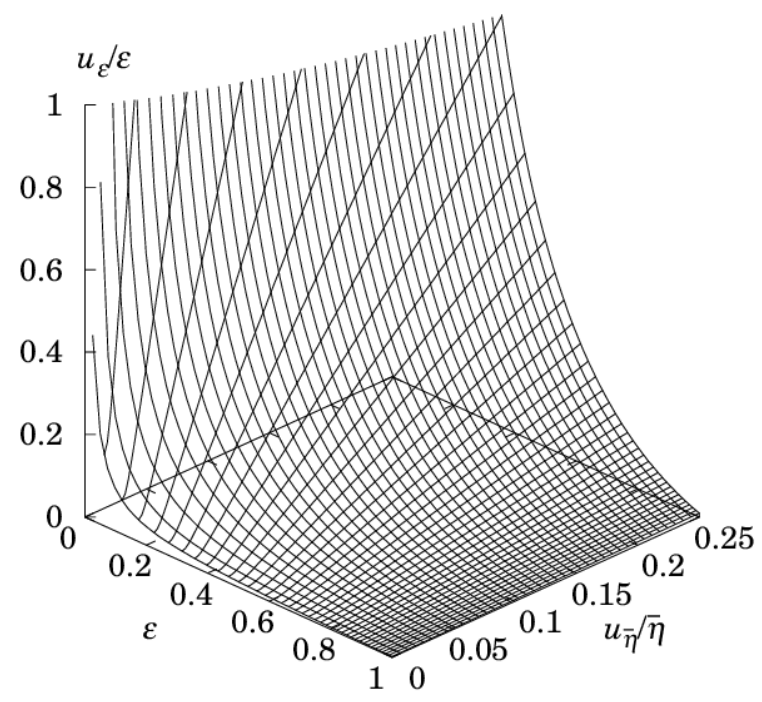

(a)

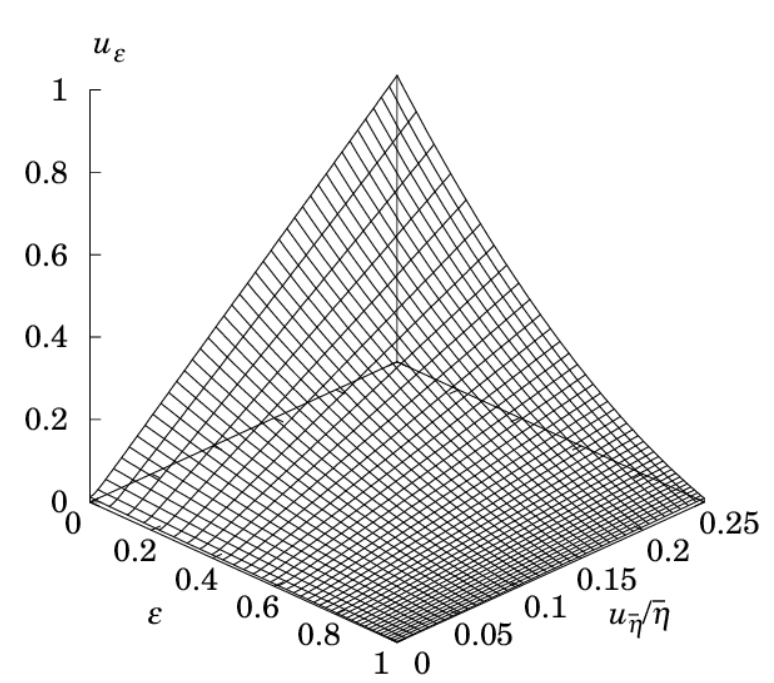

(b)

Figure 2 : Relative (a) and absolute (b) errors on porosity as function of porosity $\varepsilon$ and relative error of local backscattering signal $\frac{u_{\bar{\eta}}}{\bar{\eta}}$. Application of equation ( 9 ) with $\eta_{b}=235$,

$$
\eta_{r}=20, u_{\eta_{b}}=u_{\eta_{r}}=1 \text { and values of Table } 1
$$




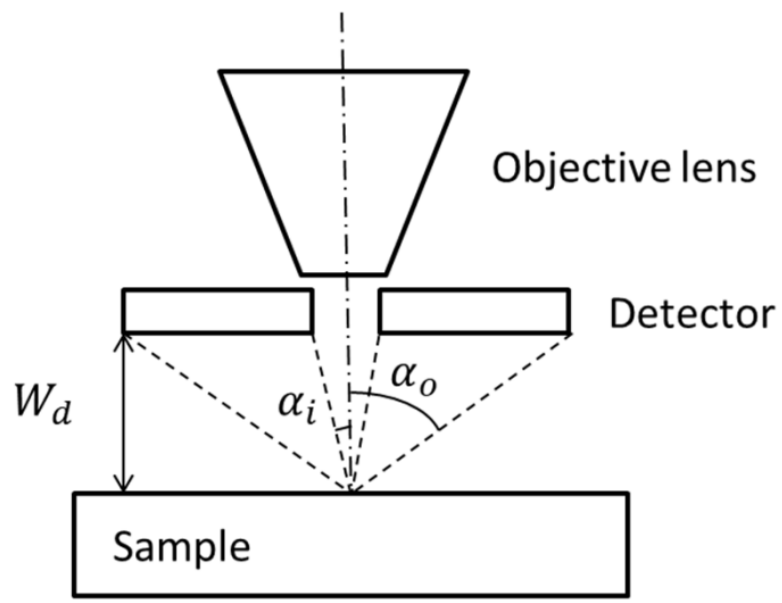

Figure 3 : Scheme of the detector geometry.

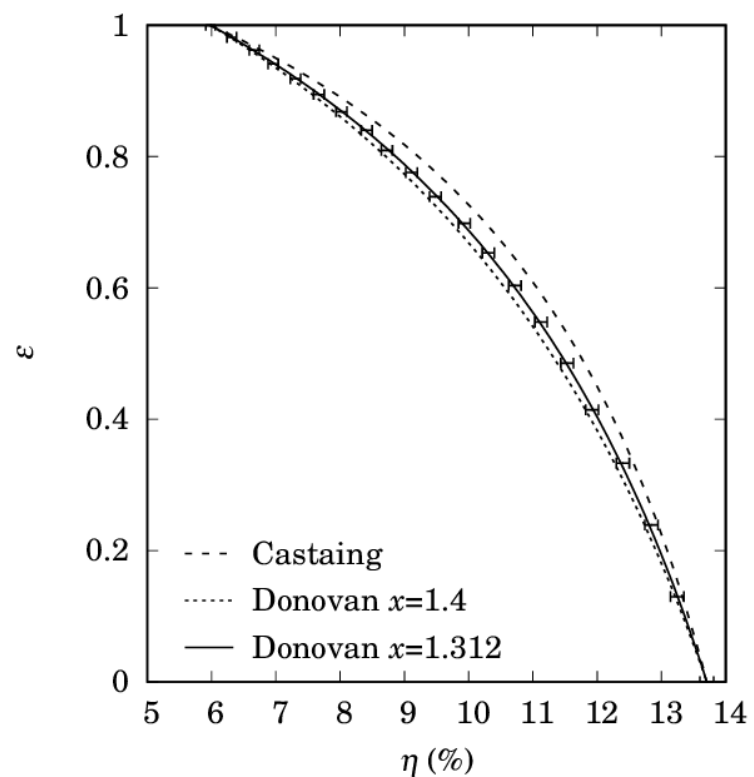

(a)

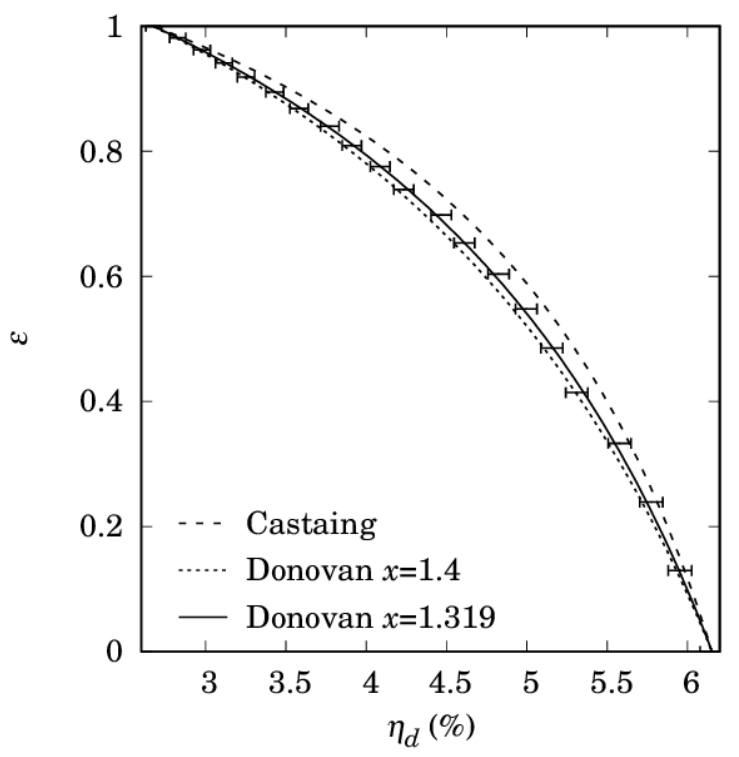

(b)

Figure 4 : Relationship between porosity $\varepsilon$ and (a) $\boldsymbol{\eta}$ total, (b) $\eta_{d}$ detected backscattering coefficients. Symbols: Monte Carlo simulations; dashed line: equation ( 5 ) Castaing relation; dotted line: equation ( 8 ) Donovan relation with exponent $x=1.4$; solid line: equation ( 8 ) Donovan relation with least-square fitted exponent $x$. 


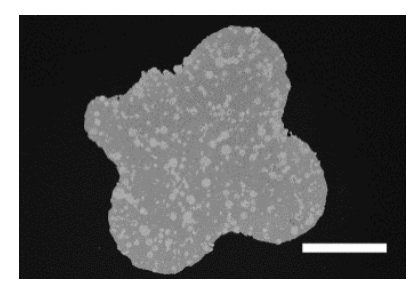

(a)

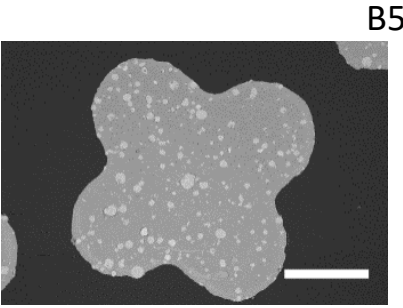

(e)

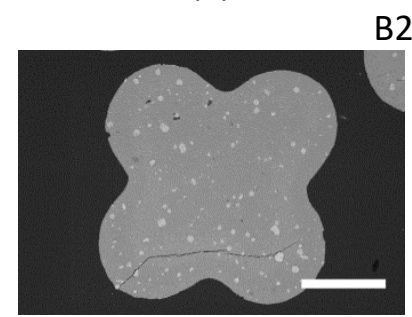

(i)

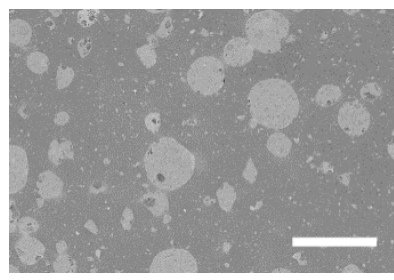

(b)

B500

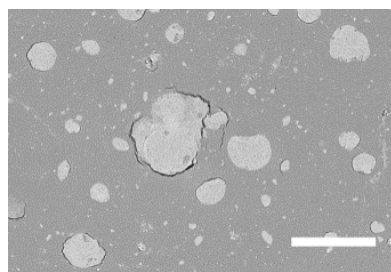

(f)

2000

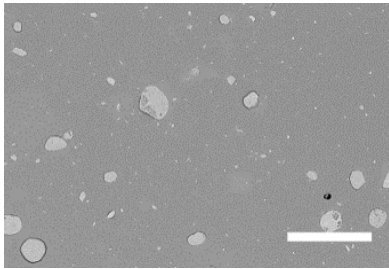

(j)

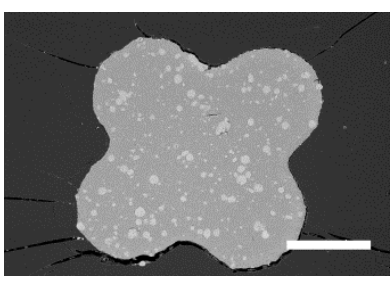

(c)

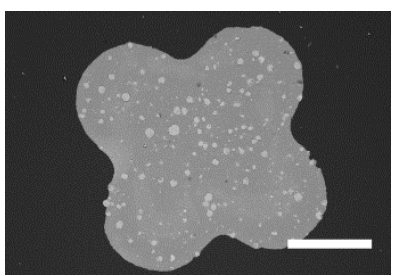

(g)

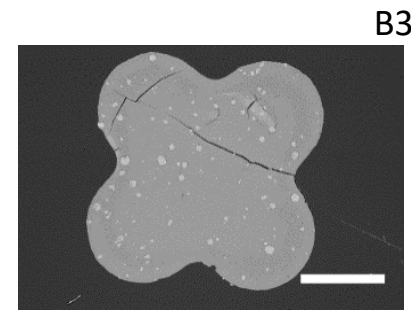

(k)

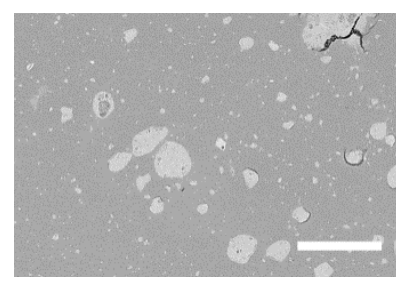

(d)

B1000

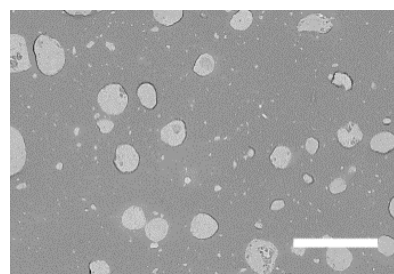

(h)

3000

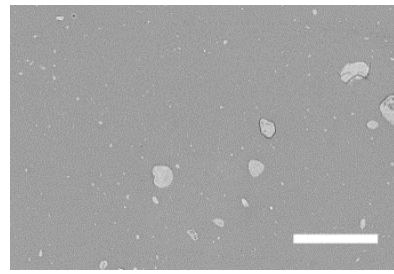

(I)

B4000 B5000

Figure 5 : Representative SEM micrograph of the alumina supports. B500 (a) (b), B1000 (c)

(d), B2000 (e) (f), B3000 (g) (h), B4000 (i) (j), B5000 (k) (l). Scale bar is 500 um for large views and $100 \mu \mathrm{m}$ for close views.

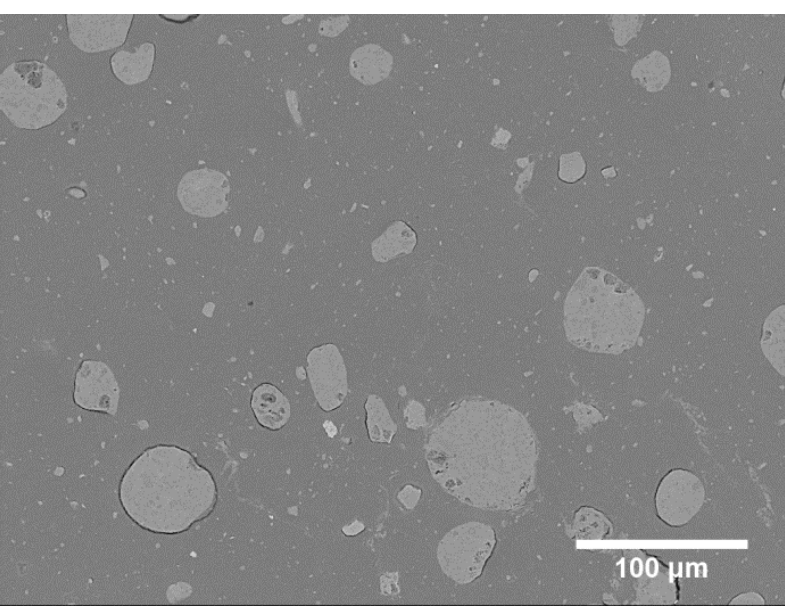

(a)

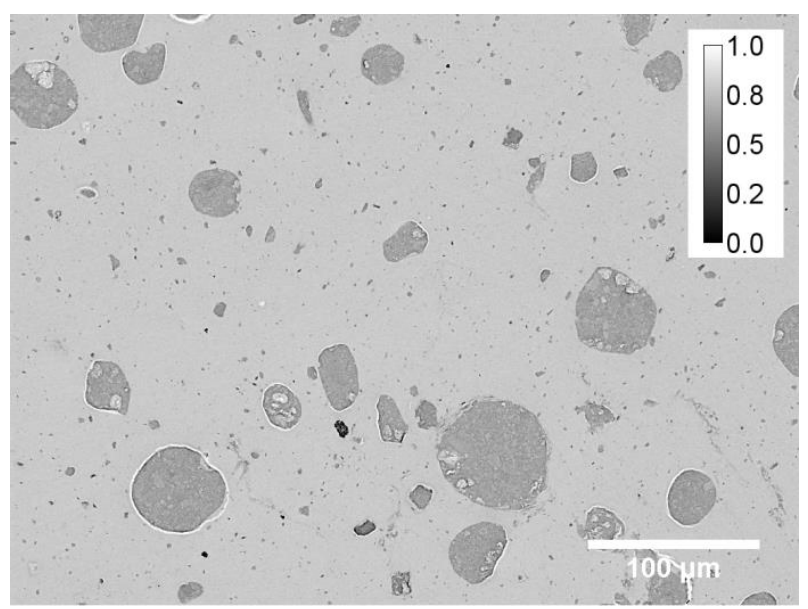

(b)

Figure 6 : Example of local porosity image of sample B2000. (a) original SEM image (b) local porosity image. 


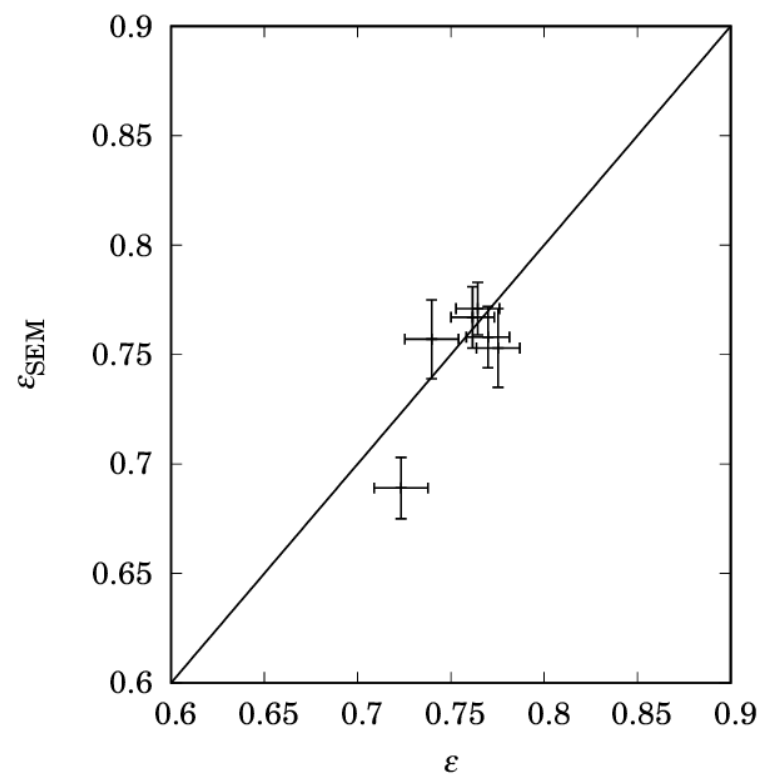

Figure 7 : Mean porosity by SEM as function of porosity from MIP. Straight line is the first bisector. 
Table 1: Physical data for Poly methyl methacrylate (PMMA) resin and alumina and estimated uncertainties. For PMMA, the molar mass is for the monomer and the specific mass for the polymer.

\begin{tabular}{|c|c|c|c|c|c|}
\hline & Formula & $\begin{array}{c}\text { Molar mass } \mathcal{M} \\
(\mathrm{g} / \mathrm{mol})\end{array}$ & $\begin{array}{c}\text { Specific mass } \rho \\
\left(\mathrm{g} / \mathrm{cm}^{3}\right)\end{array}$ & $\begin{array}{c}\text { Molar volume } \mathcal{V} \\
\left(\mathrm{cm}^{3} / \mathrm{mol}\right)\end{array}$ & $\begin{array}{c}\text { Modified atomic } \\
\text { number } Z\end{array}$ \\
\hline Alumina & $\mathrm{Al}_{2} \mathrm{O}_{3}$ & $101.96 \pm 0.01$ & $3.65 \pm 0.06$ & $27.93 \pm 0.46$ & $105.45 \pm 1.79$ \\
\hline PMMA & $\left(\mathrm{C}_{5} \mathrm{H}_{8} \mathrm{O}_{2}\right)_{\mathrm{n}}$ & $100.12 \pm 0.01$ & $1.18 \pm 0.01$ & $84.85 \pm 0.72$ & $92.15 \pm 1.15$ \\
\hline
\end{tabular}

Table 2: Textural properties of the prepared alumina supports.

\begin{tabular}{|c|c|c|c|c|c|}
\hline Sample & $\begin{array}{c}\text { Energy } \\
(\mathrm{J} / \mathrm{g})\end{array}$ & $\begin{array}{c}\rho_{g} \text { from MIP } \\
\text { volume }\left(\mathrm{g} / \mathrm{cm}^{3}\right)\end{array}$ & $\begin{array}{c}\text { Porosity from } \\
\text { MIP }\end{array}$ & $\begin{array}{c}\text { Mean porosity } \\
\text { from SEM }\end{array}$ & $\begin{array}{c}\text { Standard } \\
\text { deviation }\end{array}$ \\
\hline B500 & 500 & $1.01 \pm 0.05$ & $0.723 \pm 0.014$ & 0.689 & 0.007 \\
\hline B1000 & 1000 & $0.95 \pm 0.05$ & $0.740 \pm 0.014$ & 0.757 & 0.009 \\
\hline B2000 & 2000 & $0.87 \pm 0.04$ & $0.762 \pm 0.012$ & 0.767 & 0.007 \\
\hline B3000 & 3000 & $0.84 \pm 0.04$ & $0.770 \pm 0.012$ & 0.758 & 0.007 \\
\hline B4000 & 4000 & $0.86 \pm 0.02$ & $0.764 \pm 0.012$ & 0.771 & 0.006 \\
\hline B5000 & 5000 & $0.82 \pm 0.02$ & $0.775 \pm 0.012$ & 0.753 & 0.009 \\
\hline
\end{tabular}

